LA-UR-01-3419

Approved for public release; distribution is unlimited.

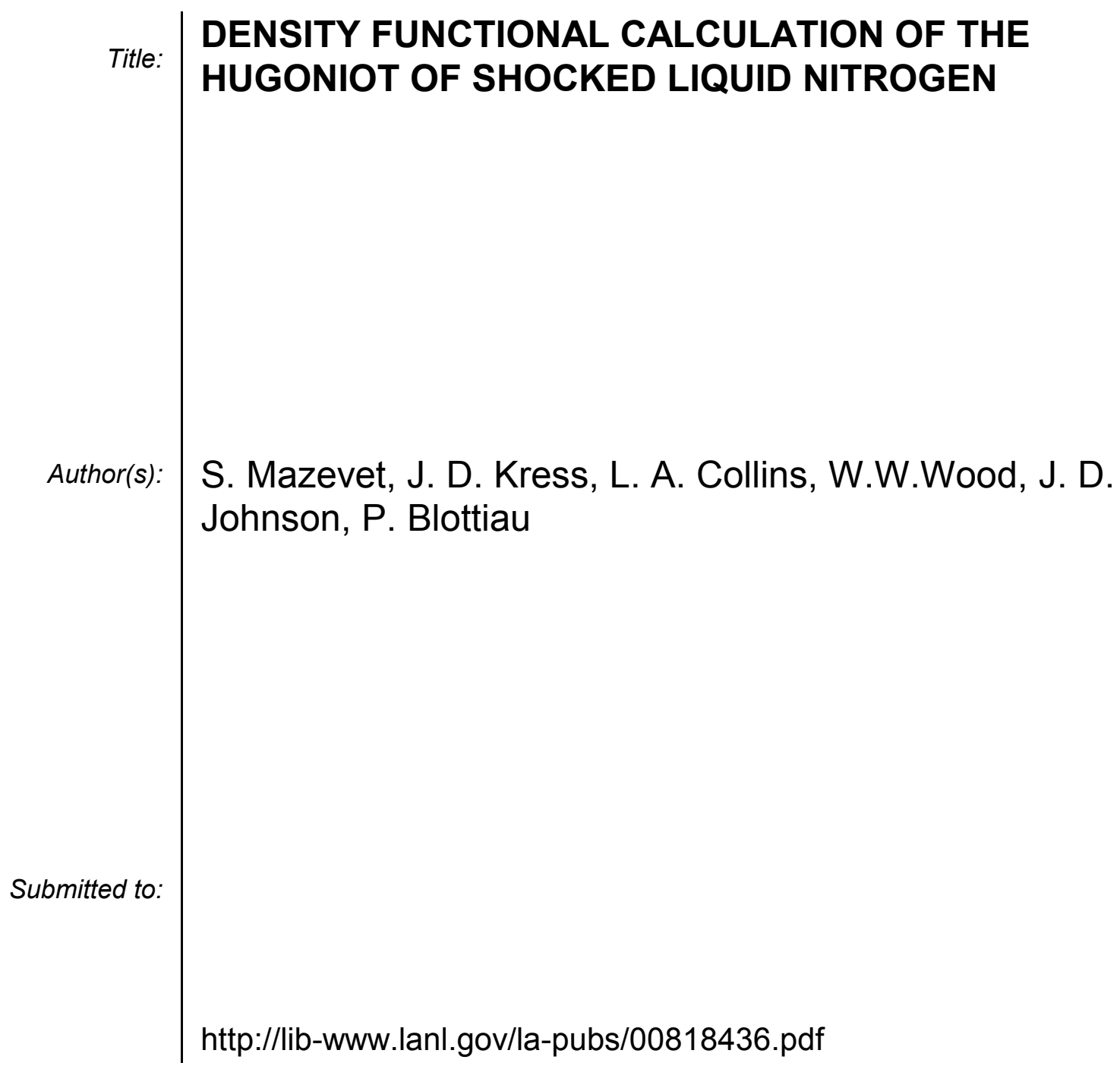

Los Alamos National Laboratory, an affirmative action/equal opportunity employer, is operated by the University of California for the U.S. Department of Energy under contract W-7405-ENG-36. By acceptance of this article, the publisher recognizes that the U.S. Government retains a nonexclusive, royaltyfree license to publish or reproduce the published form of this contribution, or to allow others to do so, for U.S. Government purposes. Los Alamos National Laboratory requests that the publisher identify this article as work performed under the auspices of the U.S. Department of Energy. Los Alamos National Laboratory strongly supports academic freedom and a researcher's right to publish; as an institution, however, the Laboratory does not endorse the viewpoint of a publication or guarantee its technical correctness. 


\title{
DENSITY FUNCTIONAL CALCULATION OF THE HUGONIOT OF SHOCKED LIQUID NITROGEN
}

\author{
S. Mazevet, ${ }^{1}$, J. D. Kress, ${ }^{1}$, L. A. Collins, ${ }^{1}$, W.W. Wood, ${ }^{1}$, J. D. Johnson,,${ }^{1}$, P. Blottiau, ${ }^{2}$ \\ ${ }^{1}$ Theoretical Division, Los Alamos National Laboratory, Los Alamos, NM 87545 \\ ${ }^{2}$ CEA, BP12 F91680, Bruyères Le Châtel, France
}

\begin{abstract}
We performed molecular dynamics simulations to obtain the internal energy and pressure of shock-compressed fluid nitrogen. Our calculations were performed using the generalized gradient approximation (GGA) in density functional theory. From this equation of state, we derived the principal and second shock Hugoniots in the density region probed by gas-gun experiments. While the single-shock Hugoniot derived from this equation of state agrees well with gas-gun experiments; in contrast, the second-shock Hugoniots show discrepancies with the experimental measurements. This is particularly the case in the region where negative Grüneisen parameters were deduced experimentally and where shock cooling was measured.
\end{abstract}

A knowledge of the properties of nitrogen, in particular the Equation-of-State(EOS), has considerable import to a wide variety of fields(1) embracing fluids, geology, shocks, detonations, and biology. A particular fertile area of investigation encompasses the behavior of fluid nitrogen under compression. Experiments $(2,3,4,5)$ have indicated that the first and second-shock Hugoniots exhibit two characteristic features, which have been attributed to the dissociation of molecular nitrogen as the increasing compression and temperature break the bonds. These feature are: 1) a slight kink in the plot of particle $\left(\mathrm{u}_{p}\right)$ verses shock $\left(\mathrm{u}_{s}\right)$ velocities for the principal Hugoniot, and 2) shock induced cooling when the material is reshocked. Renewed interest in shock-compressed nitrogen has arisen from an unlikely source, namely laser-driven shock experiments on deuterium. The close parallel between fluid deuterium, nitrogen and oxygen are reviewed elsewhere in this proceedings (6).

Over the past few years, a variety of large-scale simulation methods have been developed to treat fluid systems over a broad range of conditions. Among the most sophisticated approaches one finds the path-integral Monte Carlo (PIMC) (7) and finitetemperature density functional Molecular dynamics
(FTDFT-MD) (8) methods. In DFT approaches, the total energy is written as a functional of the electron density, which is obtained by summing the probability density over electron occupied orbitals, populated according to a Fermi-Dirac distribution at temperature $T_{e}$. Further, for GGA formulations, electronic exchange and correlation energy are approximated using a functional which depends only on the electron density and its spatial derivatives. The method encompasses all manner of transient effects such as dissociation and association of chemical bonds, quasi-molecular formation, ionization and recombination. We employed the VASP plane-wave pseudopotential code, which was developed at the Technical University of Vienna (9). This code implements the Vanderbilt ultrasoft pseudopotential scheme (10) in a form supplied by G. Kresse and J. Hafner (11) and the Perdew-Wang 91 parameterization of the GGA(12).

We performed fixed-volume molecular dynamics simulations $(13,14)$ at separate density and temperature points, selected to span a range of densities from $\rho=1.50$ to $\rho=3.35 \mathrm{~g} / \mathrm{cm}^{3}$ and temperatures from $T=750 \mathrm{~K}$ to $T=70000 \mathrm{~K}$ that highlight the first and second-shock Hugoniot region. We used 32 and 64 nitrogen atoms in the unit cell and fixed 
the plane wave cutoff at $435 \mathrm{eV}$. We employ both microcanonical and isokinetic ensembles for the ions. In the later, the ion temperature $\mathrm{T}_{i}$ is fixed using velocity scaling. In turn, the assumption of local thermodynamic equilibrium sets the electron temperature $T_{e}$ to that of the ions $T_{i}$.

The principal and multiple-shock Hugoniots satisfy the Rankine-Hugoniot equation

$$
\left(U_{0}-U_{1}\right)+\frac{1}{2}\left(V_{0}-V_{1}\right)\left(P_{0}+P_{1}\right)=0
$$

where 0 and 1 refer respectively to the initial and final conditions. $\mathrm{U}$ is the internal energy, $\mathrm{V}$ the volume, and $\mathrm{P}$ the pressure. The internal energy per atom $U$ consists of the sum of the ion kinetic energy $\left(\frac{3}{2} k_{B} T_{i}\right)$ and the time average of the DFT potential energy per ion, where $\mathrm{k}_{B}$ is the Boltzmann constant. The pressure $\mathrm{P}$ was computed in a similar fashion using the Hellmann-Feynman forces derived from the DFT potential energy. To calculate the first-shock points, we have chosen $\mathrm{P}_{0}=0, \rho_{0}=0.808$ $\mathrm{g} / \mathrm{cm}^{3}$, and $\mathrm{U}_{0}$ so that the energy of the $\mathrm{N}_{2}$ molecule is zero. The shock Hugoniot points $\left(P_{1}, T_{1}\right)$ are determined by solving Eq. (1) using a least-squares fit to a quadratic function in $\mathrm{T}$ of $\mathrm{P}$ and $\mathrm{U}$.

The DFT-MD Hugoniot points(13) appear in Fig. 1 along with the experimental data $(2,3,4)$ and the results of a chemical equilibrium model(15). The chemical model results derive from integral equation theory and Monte Carlo calculations using a classical exponential-6 pair potential. We obtain very good agreement between the DFT-MD calculations (for 32 atoms) and the experimental results all along the single-shock Hugoniot. In particular, the calculations reproduce the distinctive inflection, associated with dissociation, in the $\mathrm{u}_{s}-\mathrm{u}_{p}$ plot between particle velocities of 4 and $6 \mathrm{~km} / \mathrm{s}$. Our results support a maximum compression of around four $\left(\eta_{\max }=4\right)$. Since the experimental findings perhaps hint at a softening in the Hugoniot between $\rho=2.82$ and $2.96 \mathrm{~g} / \mathrm{cm}^{3}$ and since the chemical model definitely predicts such a trend, we have carefully examined the Hugoniot in this regime. For $\rho=3.03,3.05$ and $3.10 \mathrm{~g} / \mathrm{cm}^{3}$, DFT-MD calculations at temperatures up to $\mathrm{T}=70000 \mathrm{~K}$ and pressures up to about $300 \mathrm{GPa}$ show no solutions of Eq.1, indicating

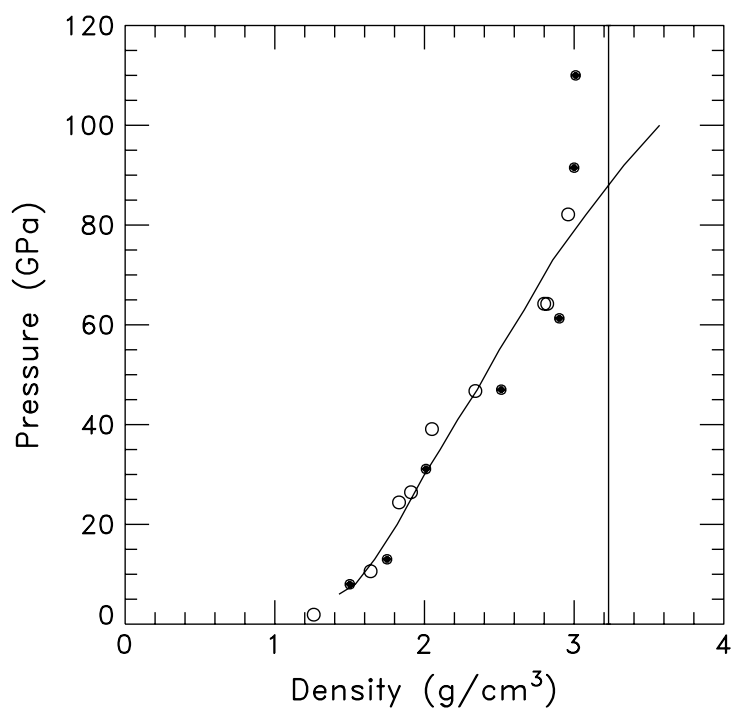

FIGURE 1. Nitrogen Hugoniot. DFT-MD (solid circles); Chemical picture (solid curve); Experiments (open circles). Vertical solid line delineates 4 fold compression.

that the pressure rises rapidly within the maximum compression line. In contrast, the chemical equilibrium model results(15) predict a softening of the Hugoniot beyond 4-fold compression.

Figure 2 shows the calculated and measured first and second-shock Hugoniot points(14). For the experimental points, the numerical labels displayed on the figure associate the first Hugoniot points to the resulting second-shock Hugoniot points when the former are re-shocked. Due to the good agreement between the first-shock GGA-MD Hugoniot and the experimental measurements in this density region, we used the experimental first-shock data of Nellis et al. (4) as initial conditions to calculate the second-shock Hugoniots. As the experimental data indicate a strong sensitivity to the initial and final states, we used, for each of the four initial experimental conditions, several of our fixed density and temperature simulations to generate the second-shock Hugoniots over a region which spans densities from $\rho=2 \mathrm{~g} / \mathrm{cm}^{3}\left(V=0.5 \mathrm{~cm}^{3} / \mathrm{g}\right)$ to $\rho=2.9 \mathrm{~g} / \mathrm{cm}^{3}\left(V=0.3448 \mathrm{~cm}^{3} / \mathrm{g}\right)$.

As seen in Fig. 2, significant disagreement 


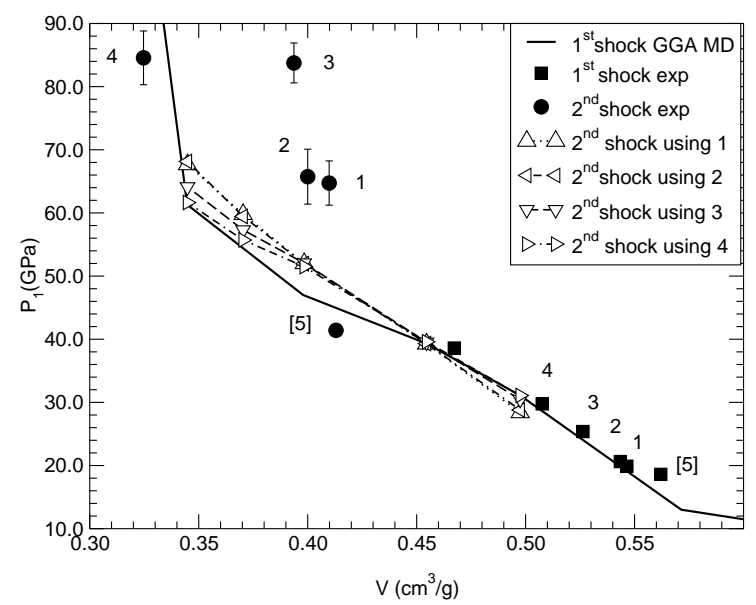

FIGURE 2. Comparison between the experimental data of ref [4] (points 1 to 4) and ref [5] (points [5]) and GGA-MD nitrogen first and second-shock Hugoniots.

exists between the predicted and measured secondshock Hugoniot points around $\rho=2.5 \mathrm{~g} / \mathrm{cm}^{3}$ $\left(V=0.4 \mathrm{~cm}^{3} / \mathrm{g}\right)$. Experimental measurements indicate second-shock pressures much higher than those of the first-shock Hugoniot when compared at fixed density. On the other hand, the GGA-MD simulations show second-shock Hugoniot points only slightly above the first-shock values in this region. The theoretical second-shock Hugoniot points have a lower pressure than the first-shock ones at the smallest density $\rho=2.0 \mathrm{~g} / \mathrm{cm}^{3}$ $\left(V=0.5 \mathrm{~cm}^{3} / \mathrm{g}\right)$, move above the first-shock Hugoniot between $\rho=2.2 \mathrm{~g} / \mathrm{cm}^{3}\left(V=0.454 \mathrm{~cm}^{3} / \mathrm{g}\right)$ and $\rho=2.9 \mathrm{~g} / \mathrm{cm}^{3}\left(V=0.344 \mathrm{~cm}^{3} / \mathrm{g}\right)$ where they cross again and drop below the principal Hugoniot. Further, while the general trend of the experimental second-shock Hugoniot points is reproduced by the GGA-MD predictions, the GGA-MD results do not show the sensitivities to the initial conditions suggested by the measurements.

At fixed density, the relative positions of the first and second-shock Hugoniot points have direct implications on the values of the Grüneisen parameter. Experimentally, a negative Grüneisen parameter was deduced in this region, an effect attributed to a transition from a molecular to an atomic fluid

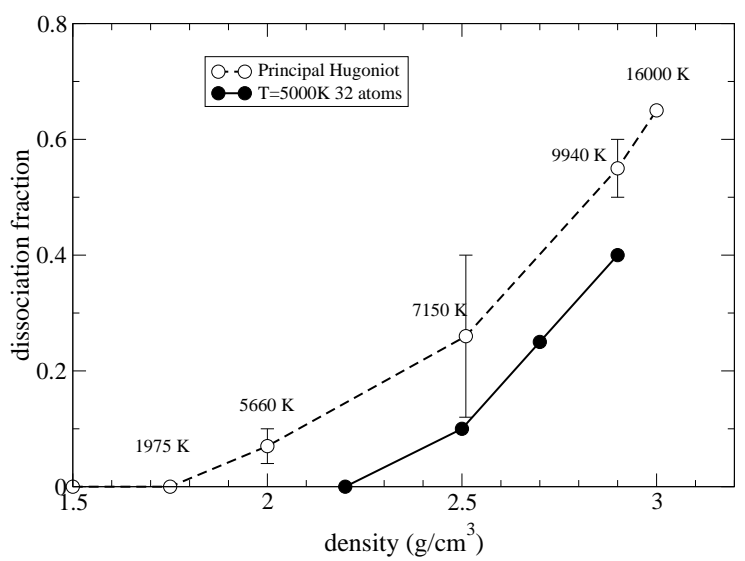

FIGURE 3. Variation of the dissociation fraction along the nitrogen principal Hugoniot and $5000 \mathrm{~K}$ isotherm as a function of the final shock density.

(3)(sometimes referred to as a "continuous phase transition"). This contrasts with our findings(14), which suggest that in the dissociating region the Grüneisen parameter is near zero, maybe slightly negative, but not as negative as the experimental measurements suggest. Further, the sensitivity in this density region to the number of atoms ( 32 vs 64 ) used in the simulation cell, also suggests that simulations for even more atoms could yield a positive value of the Grüneisen parameter.

We now turn to the actual nature of the fluid as the system passes along the Hugoniots by calculating the dissociation fraction, $\beta$. To obtain the dissociation fraction along the Hugoniots, we have performed a cluster analysis of the MD trajectories $(16,13,14)$. Figure 3 shows the variation of the dissociation fraction $\beta$, the ratio of monomers to the total number of particles obtained along the principal Hugoniot and the $5000 \mathrm{~K}$ isotherm. The latter is representative of the degree of dissociation along the second-shock Hugoniots. Fig. 3 shows that along both the first and second- shock Hugoniot, as the density, and pressure increase, the initially diatomic fluid continuously dissociates. As the fluid starts to dissociate, for densities around $2.5 \mathrm{~g} / \mathrm{cm}^{3}$, trimers and higher structures are found but appear to be highly transient, lasting for a few femtoseconds at the most. Further, as the 
compression increases, we note that the transient behavior of the fluid significantly increases as bonds break and form. The dissociation of the fluid between a density of $2.0 \mathrm{~g} / \mathrm{cm}^{3}\left(V=0.5 \mathrm{~cm}^{3} / \mathrm{g}\right)$ and $3.0 \mathrm{~g} / \mathrm{cm}^{3}\left(V=0.33 \mathrm{~cm}^{3} / \mathrm{g}\right)$ can be associated with a marked inflection of the principal Hugoniot temperature in this region(13). Further, we also find that molecular dissociation is responsible for a weak temperature variation along the second-shock Hugoniot and between the first- and second-shock Hugoniots but no significant shock cooling was observed in the simulation.

In summary, despite the excellent agreement between the GGA-MD simulation for the experimental principal Hugoniot, we find noticeable disagreement for the second-shock Hugoniots. Such discrepancies reflect upon the estimated value of the Grüneisen parameter and question the existence of the observed shock cooling. Overall, while there is qualitative agreement between the GGA-MD and the experimental measurements on the behavior of the secondshock nitrogen Hugoniot, the quantitative agreement obtained for the first shock data does not pertain for the multiple shocks.

\section{REFERENCES}

1. P. J. Kortbeekm C. A. T. Seldam, and J. A. Schouten, Mol. Phys. 69, 1001 (1990); H. D. Jones and F. J. Zerilli, J. Appl. Phys. 69, 3893 (1991); J. Touret and A. M. van den Kerkhof, Physica (Utrecht) 139, 834 (1986).

2. R. D. Dick, J. Chem. Phys. 52, 6021 (1970).

3. H. B. Radousky, W. J. Nellis, M. Ross, D. C. Hamilton, and A. C. Mitchell, Phys. Rev. Lett. 57, 2419 (1986); W. J Nellis, N. C. Holmes, A. C. Mitchell, and M. van Thiel, Phys. Rev. Lett. 53, 1661 (1984).

4. W. J. Nellis, H. B. Radousky, D. C. Hamilton, A. C. Mitchell, N. C. Holmes, K. B. Christianson, and M. van Thiel, J. Chem. Phys. 94, 2244 (1991).

5. G. L. Schott, M. S. Shaw, and J. D. Johnson, J. Chem. Phys. 82, 4264 (1985).

6. J. D. Kress, S. Mazevet, and L. A. Collins, these procedings (2001).
7. B. Militzer and D. Ceperley, Phys. Rev. Lett., 1890 (2000); B. Militzer and E. Pollock, Phys. Rev. E 61, 3470 (2000).

8. T. Lenosky, S. Bickham, J. Kress, and L. Collins, Phys. Rev. B 61, 1 (2000); G. Galli, R. Hood, A. Hazi, and F. Gygi, Phys. Rev. B 61, 909 (2000); S. Bagnier, P. Blottiau, and J. Clerouin, Phys. Rev. E 63, 015301(R) (2001).

9. G. Kresse and J. Hafner, Phys. Rev. B 47, RC558 (1993); G. Kresse and J. Furthmüller, Comput. Mat. Sci. 6, 15-50 (1996); G. Kresse and J. Furthmüller, Phys. Rev. B 54, 11169 (1996).

10. D. Vanderbilt, Phys. Rev. B 41, 7892 (1990).

11. G. Kresse and J. Hafner, J. Phys. Condens. Matter 6, 8245 (1994).

12. J. P. Perdew, in Electronic Structure of Solids, ed. by F. Ziesche and H. Eschrig (Akademie Verlag, Berlin, 1991).

13. J. D. Kress, S. Mazevet, L.A . Collins, and W. W. Woods, Phys. Rev. B 63, 024203 (2001).

14. S. Mazevet, J. D. Johnson, J. D. Kress, L. A. Collins, and P. Blottiau, Phys. Rev. B (to be published).

15. L. E. Fried and W. M. Howard, J. Chem. Phys. 109, 7338 (1998).

16. T. J. Lenosky, J. D. Kress, L. A. Collins, and I. Kwon, Phys. Rev. B 55, R11907 (1997);T. J. Lenosky, J. D. Kress, and L. A. Collins, Phys. Rev. B 56, 5164 (1997); T. J. Lenosky, J. D. Kress, L. A. Collins, and I. Kwon, J. Quant. Spectrosc. Radiat. Transfer 58, 743 (1997). 